\title{
An 11b 60MS/s 2.1mW Two-Step Time-Interleaved SAR-ADC with Reused S\&H
}

\author{
Sai-Weng Sin, Li Ding, Yan Zhu, He-Gong Wei, Chi-Hang Chan, U-Fat Chio, \\ Seng-Pan U, R. P. Martins ${ }^{1}$, Franco Maloberti ${ }^{2}$ \\ Analog and Mixed Signal VLSI Laboratory (http://www.fst.umac.mo/en/lab/ans_vlsi/index.html) \\ Faculty of Science and Technology, University of Macau, Macao, China \\ Tel:+853 83978795, Fax: +853 28840542, email: terryssw@umac.mo \\ 1 - On leave from Instituto Superior Técnico/TU of Lisbon, Portugal \\ 2 - University of Pavia, Italy
}

\begin{abstract}
An 11b 60MS/s 2-channel two-step SAR ADC in $65 \mathrm{~nm}$ CMOS is presented. The scheme shares the op-amp between channels for the residual generation and takes advantage of time interleaving for reusing the input $\mathrm{S \& H}$ of the first stage. A reduction of the gain in the residual generator and subthreshold operation enables the use of a power-effective, singlestage op-amp with 69dB-gain. The ADC achieves peak SNDR of $57.6 \mathrm{~dB}$ while consuming $2.1 \mathrm{~mW}$ from $1-\mathrm{V}$ analog and $0.85-\mathrm{V}$ digital supply, resulting in an FoM of $57 \mathrm{fJ} /$ step.
\end{abstract}

\section{INTRODUCTION}

The technology scaling favors the design of ultra power efficient, moderate resolution and high-speed successive approximation register (SAR) ADCs [1]-[3]. The power effectiveness of traditional pipelined or two-step ADCs drops for $10+$ bit and 50-100 MS/s specifications because of the power required by the op-amp(s). The problem becomes more evident with nanometer CMOS technologies because highgain op-amps are difficult to design. However, high resolution and high-speed need amplification, and consequently, the use of op-amps become unavoidable. Published results show that with low or medium resolution (less than 10-bit) the SAR architecture obtains excellent figures of merit with conversion speeds around $50 \mathrm{MS} / \mathrm{s}$. However, when the resolution goes above 10-bit and the speed is several tens of $\mathrm{MHz}$ the power performances drop sharply, even because of the power needed by the reference voltage generator. Accordingly, sustaining the figure of merit at state-of-the-art levels become problematic.

This work addresses the problem of obtaining power effectiveness in the $10+$ bit and $50+\mathrm{MS} / \mathrm{s}$ regions. The used solution obtains the conversion in two-steps but uses 6-bit SARs instead than flash schemes because the SAR is much more power effective. The interstage gain is 8 . Therefore, the

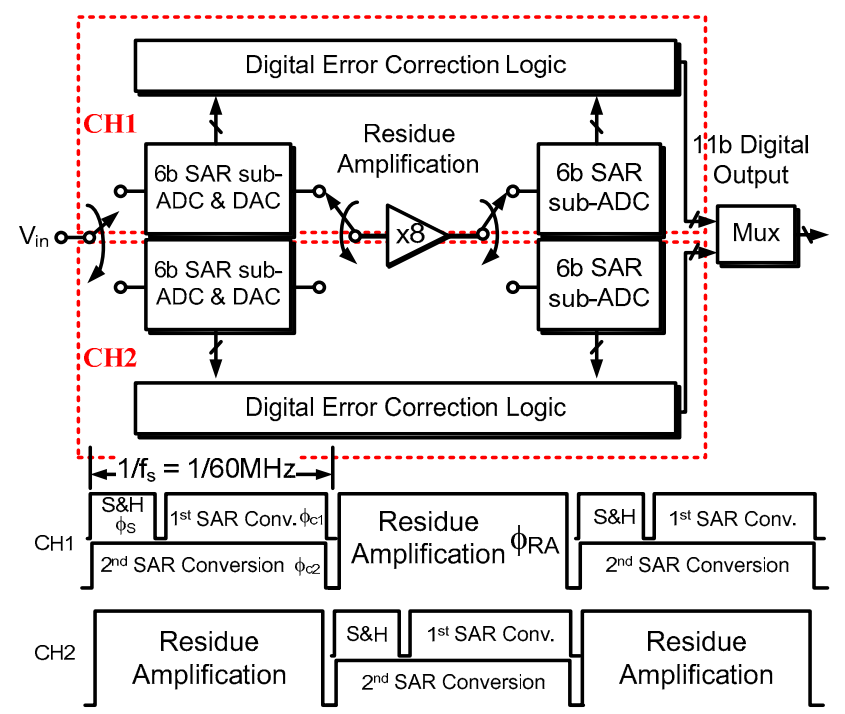

Fig. 1. The overall ADC architecture and timing diagram.

obtained low swing of the residual generator enables using a telescopic gain-boosted cascode, shared between timeinterleaved paths. The digital error correction relaxes the $1 \mathrm{st}$ SAR requirements. All the above mentioned methods permit us to obtain a very low FoM, making the proposed scheme a valid and power effective alternative to single-step dynamic SAR ADC whose optimal region of operation is in the lower resolution and higher speed range.

\section{The Proposed ADC ARChiteCtURE}

Fig. 1 shows the overall $11 \mathrm{~b} 60 \mathrm{MS} / \mathrm{s}$ ADC architecture that uses two interleaved two-step SAR ADCs sharing the same residue amplifier. The $1^{\text {st }}$ stage converts the coarse $6 \mathrm{~b}$ 


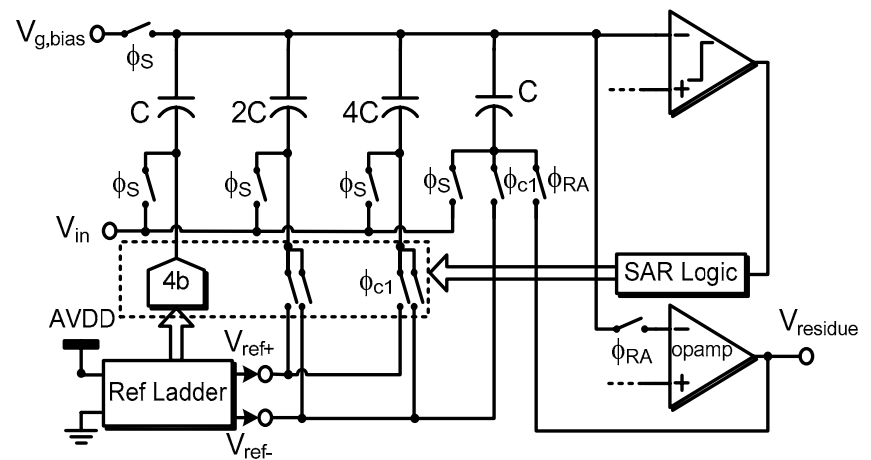

Fig. 2. The $1^{\text {st }}$-stage SAR followed with $8 \mathrm{x}$ residue amplification.

codes and generates the residue, which is amplified by 8 and it is passed to the $2^{\text {nd }} \mathrm{SAR}$ sub-ADC which converts $6 \mathrm{~b}$ fine codes. The digital correction logic uses coarse and fine codes to obtain $11 \mathrm{~b}$. The $\mathrm{V}_{\text {ref }}= \pm 500 \mathrm{mV}$ is generated by an on-chip reference ladder. Fig. 1 also shows timing diagram and clock phases.

The gain of the residue amplifier should be normally 32 to comply with the $6 \mathrm{~b}$ of the $1^{\text {st }}$ stage. However, the low feedback factor would lead to expensive requirement of high DC open loop gain and high op-amp's GBW. Moreover, high output swing in the op-amp would constrain the low-voltage op-amp architecture. A suitable trade-off is to use a gain of 8 , which leads to an affordable request of $2^{\text {nd }}$-stage accuracy equal to $8 \mathrm{~b}$. The benefit is that the op-amp gain, the speed and swing requirements are all relaxed with respect to what the $32 \mathrm{x}$ amplification needs.

Fig. 2 shows the block diagram of the $1^{\text {st }} 6 \mathrm{~b}$ stage and the residue generator. An array of 8 unity capacitors and a $4 \mathrm{~b}$ resistive ladder make the $6 \mathrm{~b}$ DAC. A process-tracked biasing network generates $\mathrm{V}_{\mathrm{g} \text {,bias }}$ for setting the proper value of the input common-mode voltage. The DAC array is pre-charged (with bootstrapped switches) to the input signal during $\phi_{S}$ (specified in the timing of Fig. 1). Then, a $6 \mathrm{~b}$ self-timing SAR converter works during the remaining time-slot $\phi_{\mathrm{Cl}}$. At the end of the conversion the residue charge remains on the top-plate of the DAC array. That charge is integrated on a unity capacitance of the array that operates as a flip-around element for obtaining the $8 \mathrm{x}$ residual generation during $\phi_{\mathrm{RA}}$. The scheme shares the operational amplifier between the two timeinterleaved paths. The time-interleaving enables a double use of the capacitive array: to perform the input sampling and the generation of the residual. Therefore, the signal sampled for coarse conversion is reused for the residual generation. This feature reduces the input capacitive load and avoids error caused by clock misalignment in sampling the input for the coarse and the fine conversions. Moreover, the use of timeinterleave reduces the operating frequency in each path, but the path mismatches including offset, gain and timing will

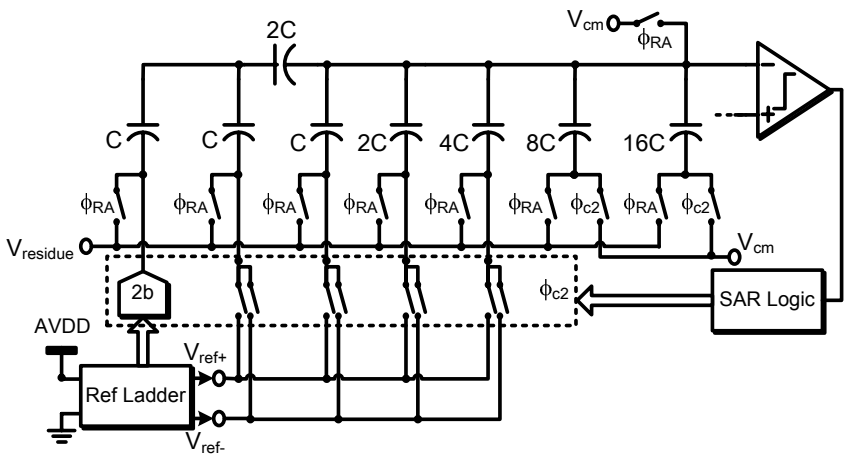

Fig. 3. The $2^{\text {nd }}$-stage SAR sub-ADC.

create problems. For improving performances, the $\mathrm{S} / \mathrm{H}$ reuse allows the use of bigger unity capacitances that improve the matching.

The fine SAR conversion with self-timing operation occurs in a pipeline fashion while the first stage starts a new cycle. Fig. 3 shows the architecture of the $2^{\text {nd }}$ stage sub-ADC. It is composed by a $4 \mathrm{~b}$ capacitive and a $2 \mathrm{~b}$ resistive array. The capacitors $8 \mathrm{C}$ and $16 \mathrm{C}$ are not part of the sub-ADC but perform a scaling by 4 of the references $( \pm 125 \mathrm{mV})$ as required by the lower interstage gain, which is 8 and not 32. The used solution enables the utilization in the fine-bit DAC reference voltages that are equal to the one employed in the coarse-bit DAC.

A master clock of $60 \mathrm{MHz}$ generates various matched interleaved clock phases through a divider-by-2 low-skew clock generator. The self-timed SAR logic avoids the need of external fast master clock. All SAR loop utilized dynamic logics to enable high speed processing, with trade-offs of possible leakages that degrade the digital logic performance at low sampling frequencies.

The use of digital error correction relaxes the accuracy of the $1^{\text {st }}$ stage sub-ADC decisions (including the ones related to the comparator offset, the noise and incomplete DAC settlings during the SAR's bit-cycling) from $11 \mathrm{~b}$ to only $7 \mathrm{~b}(7.8 \mathrm{mV})$. Indeed, the $1^{\text {st }}$-stage operation is required to settle within the needed accuracy only at the end of the residue amplification phase. The time allocated for this phase is almost half of the clock period and the output voltage is within $\pm 125 \mathrm{mV}$; therefore, time-constant of the $1^{\text {st }}$ stage ladder and specifications of the op-amp are not problematic.

The $1^{\text {st }}$-stage comparator calibrates the offset through an unbalanced capacitive loading technique [4]. Since the offset auto-zeroing for the op-amp would require an additional clock phase, the design obtains a $3 \sigma$ offset of $4.5 \mathrm{mV}$ by using a large input differential pair operating in the sub-threshold region. The $2^{\text {nd }}$ stage comparator offset is cancelled in the digital domain by interleavely subtracting the mean code. 


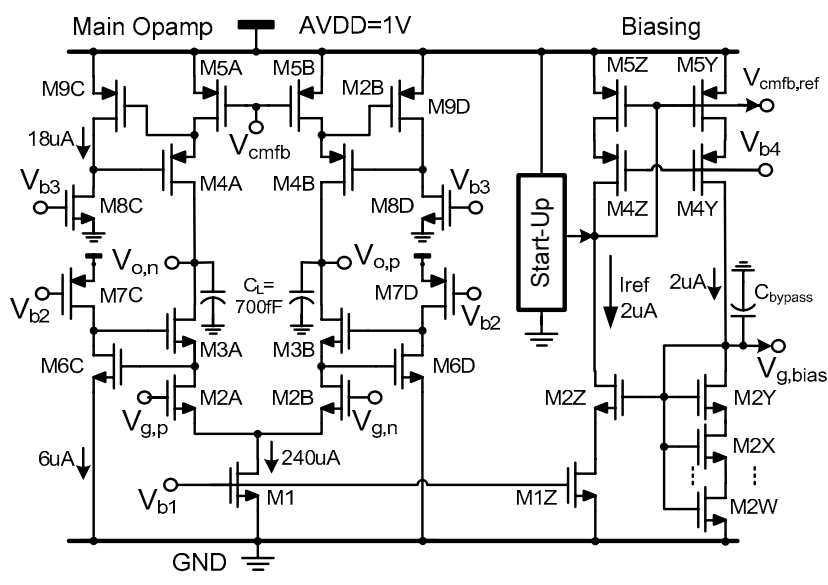

Fig. 4. Circuit schematic of the telescopic gain-boosting op-amp.

\section{THE SUB-THRESHOLD OPAMP}

Fig. 4 shows the circuit schematic of the single-stage opamp along with its biasing circuit able to track process variations. The bias network also generates $\mathrm{V}_{\mathrm{g} \text {,bias }}$ for the proper setting the op-amp input common-mode. The start-up circuit ensures non-zero current in the bias network. Conventional op-amps designed in low-voltage nanometer CMOS technologies need to use two-stage architectures. This work, thanks to the reduced output swing, can use a telescopic configuration with a gain-boosting technique that enhances the gain. The used currents keep transistors in the sub-threshold, thus minimizing overheads and making possible a 1-V supply. Thanks to sub-threshold conditions, the $\mathrm{V}_{\mathrm{GS}}$ of transistors M9C can be set quite close to the overdrive voltage $\mathrm{V}_{\mathrm{OD}}$ of M5A. This feature, made possible by the sub-threshold operation and a nanometer technology, does not increase the headroom needed by the gain-boosted telescopic op-amp which was the case in older technology node. Since the opamp gain is in the order of $\left(\mathrm{g}_{\mathrm{m}} \mathrm{r}_{\mathrm{o}}\right)^{3}$, the transistor lengths can be reduced significantly to alleviate the large aspect-ratio requirements of the sub-threshold transistors. The gainboosting branches consume $48 \mu \mathrm{A}$ (that is $16 \%$ of $300 \mu \mathrm{A}$ the total current of the op-amp). The op-amp achieves $69 \mathrm{~dB}$ gain, $440 \mathrm{MHz}$ GBW and $88^{\circ}$ phase margin with $\beta=1 / 8$ with the use of standard- $\mathrm{V}_{\mathrm{T}}$ transistors.

\section{MeAsurement Results}

The prototype ADC was implemented in a $65 \mathrm{~nm}$ standard CMOS process. Fig. 5 shows the die photograph whose core area is $0.21 \mathrm{~mm}^{2}$. The ADC has a full-scale input range of $1 \mathrm{~V}_{\mathrm{pp}}$ differential. Fig. 6 reports the static linearity. The DNL and INL are 0.39/-0.34 LSB and 1.4/-1.3 LSB, respectively, showing the expected $11 \mathrm{~b}$ performance at low frequency. However, similarly to already published experimental results, dynamic limitations cause harmonic components as shown in the output spectrum of Fig. 7. The measured FFT plot with

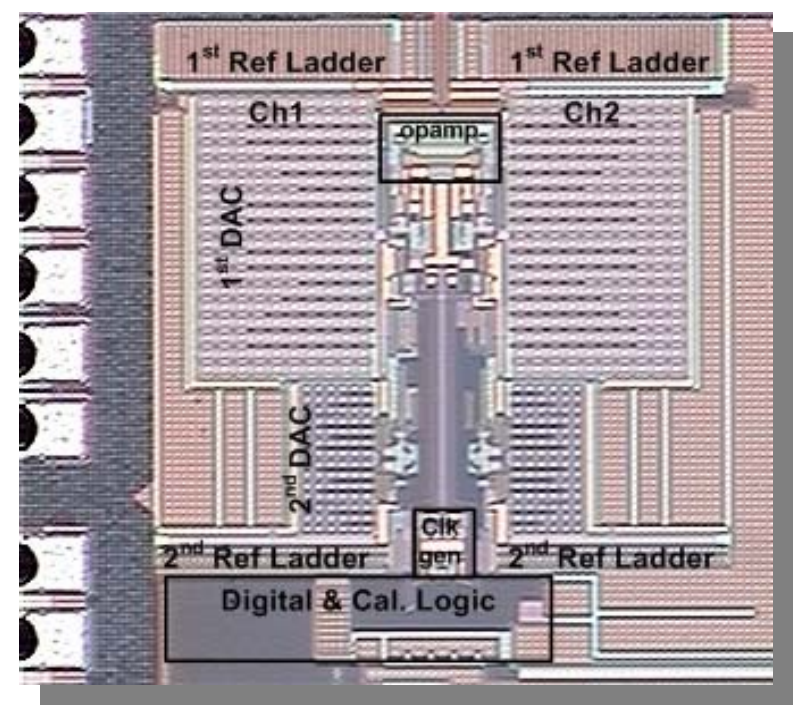

Fig. 5: Chip micrograph.

$2.3 \mathrm{MHz}$ input and $60 \mathrm{MHz}$ sampling frequency indicates SNDR/SFDR/THD equal to $57.6 / 63 /-61 \mathrm{~dB}$, respectively. As illustrated in Fig. 8, inputs in the $2-10 \mathrm{MHz}$ range show an SNDR of about $57 \mathrm{~dB}$. The SNDR drops at low $f_{s}$ (Fig. 9) because of the leakage in the dynamic SAR logic. The expected limit is irrelevant because this design is for $f_{s}>50$ $\mathrm{MHz}$. The power consumption is $2.1 \mathrm{~mW}$ with $1-\mathrm{V}$ analog and $0.85-\mathrm{V}$ digital supply. The analog power, including reference ladders $(1.3 \mathrm{~mW})$, op-amp $(300 \mu \mathrm{W})$, and comparators $(100 \mu \mathrm{W})$, is $1.7 \mathrm{~mW}$. The digital consumed power is $0.4 \mathrm{~mW}$. The performance comparison of the ADC to the state-of-theart designs is given in Table I.

\section{CONCLUSIONS}

This $11 \mathrm{~b} 60 \mathrm{MS} / \mathrm{s}$ time-interleaved two-step SAR ADC proposes and demonstrates various methods for achieving the specifications with very low power. Sub-threshold operation and a reduced output swing allow an effective design of the needed high-gain low-power op-amp. The design achieved a significant FoM of $57 \mathrm{fJ} /$ conv, comparable to state-of-the art fully-dynamic SAR ADCs.

\section{ACKNOWLEDGMENT}

This work was financially supported by the Research Grant of University of Macau and Macao Science and Technology Development Fund (FDCT) with Ref no: RGUL/07-08S/Y1/MR01/FST and FDCT/009/2007/A1.

\section{REFERENCES}

[1] C.C. Liu, et Al. "A $0.92 \mathrm{~mW}$ 10-bit 50-MS/s SAR ADC in 0.13 $\mu \mathrm{m}$ CMOS Process," in Symp. VLSI Circuits Dig. Tech. Papers, pp.236-237, Jun. 2009.

[2] M. Yoshioka, et Al. "A 10b 50MS/s $820 \mu \mathrm{W}$ SAR ADC with On-Chip Digital Calibration," IEEE ISSCC Dig. Tech. Papers, pp. 384-385, Feb. 2010. 
[3] Yan Zhu, et Al. " A 10-bit 100-MS/s Reference-Free SAR ADC in 90nm CMOS," in IEEE Journal of Solid-State Circuits, vol. 45, no. 6, pp. 1111 - 1121, Jun 2010.

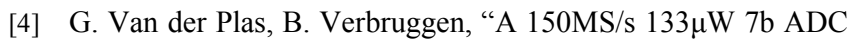
in $90 \mathrm{~nm}$ digital CMOS Using a Comparator-Based Asynchronous Binary-Search sub-ADC," ISSCC Dig. Tech. Papers, pp. 242-243, 2008.
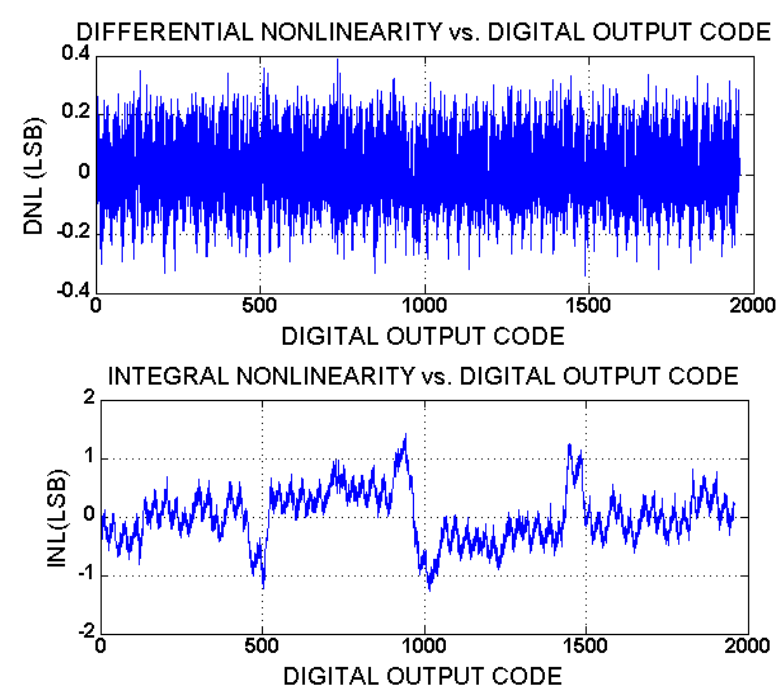

Fig. 6. Measured DNL and INL of the ADC.

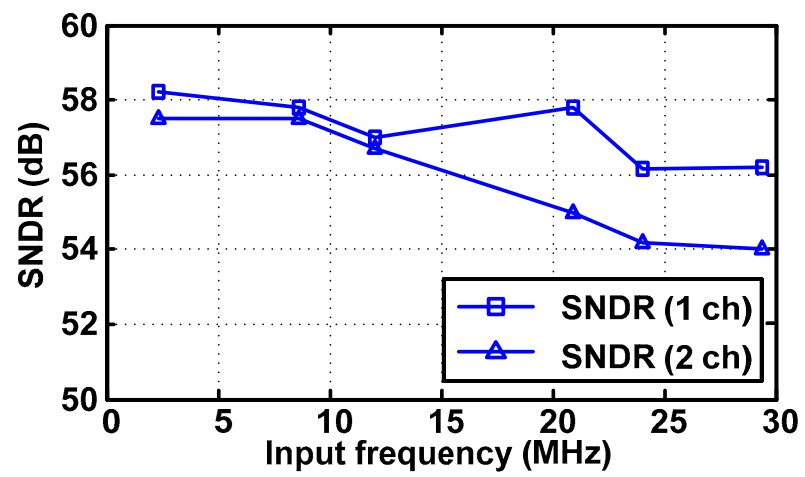

Fig. 8. SNDR vs. the input frequency.
[5] Jae-Won Nam, Young-Deuk Jeon, Young-Kyun Cho, SangGug Lee and Jong-Kee Kwon, "A 2.85mW 0.12mm2 1.0V 11bit 20-MS/s Algorithmic ADC in 65nm CMOS," in Proc. ESSCIRC, pp. 468 - 471, Sept. 2009.

[6] L. Brooks, Hae-Seung Lee, "A 12b 50MS/s Fully Differential Zero-Crossing-Based ADC Without CMFB," ISSCC Dig. Tech. Papers, pp. 166-167, Feb. 2009.

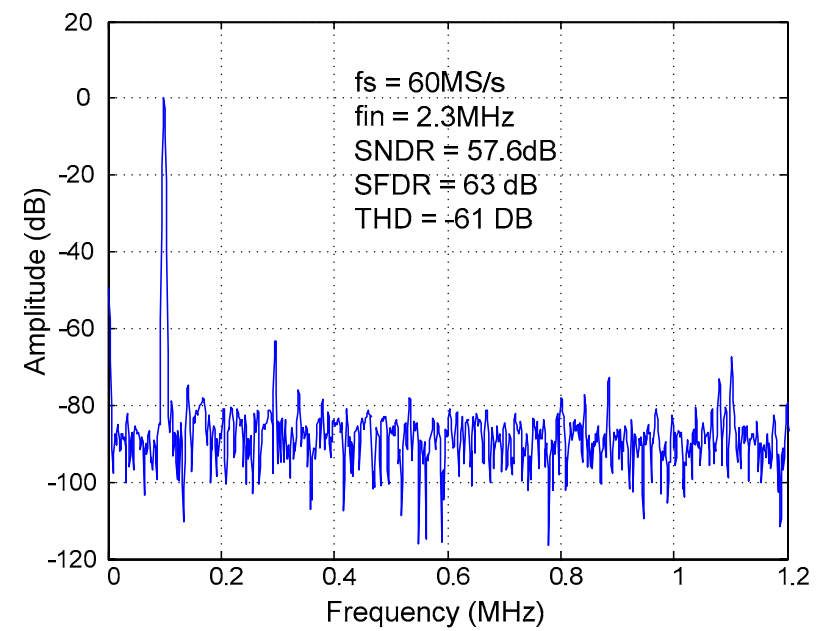

Fig. 7. Measured output spectrum of the $\mathrm{ADC}$ with $f_{s}=60 \mathrm{MS} / \mathrm{s}$ (Output decimated by 25)

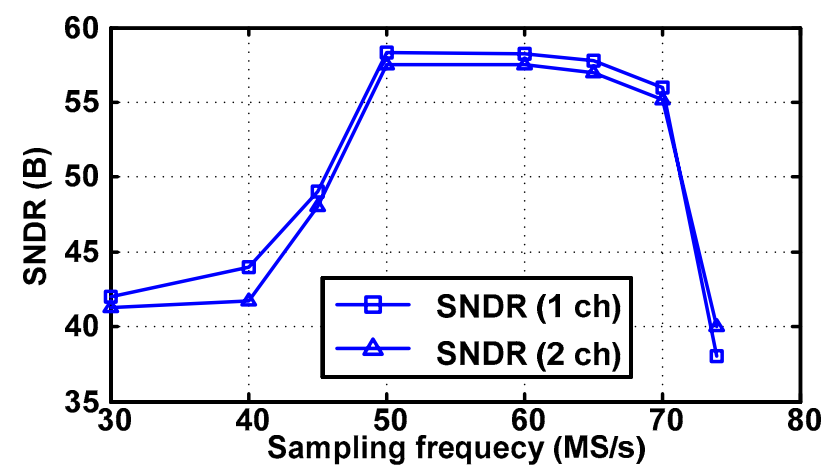

Fig. 9. SNDR vs. the sampling frequency.

TABLE I: Comparison to the state-of-the-art ADCs.

\begin{tabular}{|c|c|c|c|c|c|c|}
\cline { 2 - 7 } \multicolumn{1}{c|}{} & {$[1]$ VLSI'09 } & [2] ISSCC'10 & [3] JSSC'10 & [5] ESSCIRC'09 & [6] ISSCC'09 & This Work \\
\hline CMOS Technology & $0.13 \mu \mathrm{m}$ & $65 \mathrm{~nm}$ & $65 \mathrm{~nm}$ & $65 \mathrm{~nm}$ & $90 \mathrm{~nm}$ & $65 \mathrm{~nm}$ \\
\hline Architecture & SAR & SAR & SAR & Algorithmic & Pipelined & Tl-Two-Step \\
\hline Opamp use & No & No & No & Yes & $\begin{array}{c}\text { Zero-Crossing- } \\
\text { Based }\end{array}$ & Yes \\
\hline Resolution & $10 \mathrm{~b}$ & $10 \mathrm{~b}$ & $10 \mathrm{~b}$ & $11 \mathrm{~b}$ & $12 \mathrm{~b}$ & $11 \mathrm{~b}$ \\
\hline Sampling Rate & $50 \mathrm{MS} / \mathrm{s}$ & $50 \mathrm{MS} / \mathrm{s}$ & $100 \mathrm{MS} / \mathrm{s}$ & $20 \mathrm{MS} / \mathrm{s}$ & $50 \mathrm{MS} / \mathrm{s}$ & $60 \mathrm{MS} / \mathrm{s}$ \\
\hline Power & $920 \mu \mathrm{W}$ & $820 \mu \mathrm{W}$ & $3 \mathrm{~mW}$ & $2.85 \mathrm{~mW}$ & $4.5 \mathrm{~mW}$ & $2.1 \mathrm{~mW}$ \\
\hline DNL (LSB) & $0.88 /-1$ & 0.7 & $0.79 /-0.27$ & $0.37 /-0.32$ & $0.68 /-0.4$ & $0.39 /-0.34$ \\
\hline INL (LSB) & $2.2 /-2.1$ & 0.82 & $0.86 /-0.78$ & $0.72 /-0.64$ & $2.7 /-2.7$ & $1.4 /-1.3$ \\
\hline SNDR & $52.8 \mathrm{~dB}$ & $56.9 \mathrm{~dB}$ & $56.6 \mathrm{~dB}$ & $60.4 \mathrm{~dB}$ & $62 \mathrm{~dB}$ & $57.6 \mathrm{~dB}$ \\
\hline ENOB & 8.5 & 9.2 & 9.1 & 9.7 & 10 & 9.3 \\
\hline FoM (P/[2 ${ }^{\text {ENOB }}{ }_{*}$ fs] $]$ & $52 \mathrm{fJ} / \mathrm{step}$ & $30 \mathrm{fJ} / \mathrm{step}$ & $55 \mathrm{fJ} / \mathrm{step}$ & $167 \mathrm{fJ} / \mathrm{step}$ & $\mathbf{8 8 f J / s t e p}$ & $57 \mathrm{fJ} / \mathrm{step}$ \\
\hline
\end{tabular}

